\title{
La prévention des troubles musculo-squelettiques en conception : quelles marges de manœuvre pour le déploiement de l'activité ? de Coutarel
}

\section{Sandrine Caroly}

\author{
(2) OpenEdition \\ Journals \\ Édition électronique \\ URL : http://journals.openedition.org/pistes/3778 \\ DOI : $10.4000 /$ pistes.3778 \\ ISSN : 1481-9384 \\ Éditeur \\ Les Amis de PISTES
}

Édition imprimée

Date de publication : 1 mai 2006

\section{Référence électronique}

Sandrine Caroly, «La prévention des troubles musculo-squelettiques en conception : quelles marges de manœuvre pour le déploiement de l'activité ? de Coutarel », Perspectives interdisciplinaires sur le travail et la santé [En ligne], 8-1 | 2006, mis en ligne le 01 mai 2006, consulté le 22 septembre 2020. URL : http://journals.openedition.org/pistes/3778; DOI : https://doi.org/10.4000/pistes.3778

Ce document a été généré automatiquement le 22 septembre 2020.

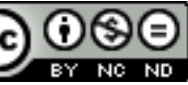

Pistes est mis à disposition selon les termes de la licence Creative Commons Attribution - Pas d'Utilisation Commerciale - Pas de Modification 4.0 International. 


\title{
La prévention des troubles musculo- squelettiques en conception : quelles marges de manœuvre pour le déploiement de l'activité ? de Coutarel
}

\author{
Sandrine Caroly
}

\section{RÉFÉRENCE}

Coutarel, F. (2004). La prévention des troubles musculo-squelettiques en conception : quelles marges de manœuvre pour le déploiement de l'activité ? Thèse de doctorat en ergonomie, Université Victor Segalen Bordeaux 2, Éditions du Laboratoire d'Ergonomie des Systèmes Complexes

1 Cette thèse soutenue au Laboratoire d'Ergonomie des Systèmes Complexes par Fabien Coutarel situe l'approche ergonomique de la prévention des TMS, problème de santé au travail pour les salariés touchés par ce phénomène de plus en plus préoccupant pour les entreprises et les pouvoirs publics. Après une approche historique et une analyse des enjeux permettant de situer et de dater la problématique des TMS, cette thèse définit bien le point de vue de l'ergonomie. Les TMS sont considérés comme une pathologie de l'organisation en lien avec la diminution des marges de manœuvre pour les opérateurs et pour l'encadrement. La méthodologie pour prévenir les TMS dans le travail répétitif consiste à mettre en œuvre une conduite de projet de conception méthodologie illustrée dans cette thèse par une belle intervention dans un atelier de découpe de canard.

2 Cette thèse met l'accent sur un modèle de la santé, mais surtout sur l'exploration en matière d'efficacité du travail et se demande de quels repères méthodologiques on 
dispose pour mener une intervention ergonomique visant à prévenir les atteintes à la santé, et plus particulièrement, la survenue des TMS.

Deux perspectives étiologiques principales sont mobilisées :

- les TMS ne sont pas une pathologie des personnes mais un «syndrome d'une organisation du travail déficiente » (p.181);

- les TMS sont le reflet d'un déficit de marges de manœuvre des salariés dans leur travail.

Dans ce contexte, F. Coutarel souligne l'intérêt de concevoir la prévention des TMS autour de trois orientations :

- l'augmentation des marges de manœuvre des opérateurs et de l'encadrement ;

- l'intervention ergonomique centrée sur les marges de manœuvre considère simultanément les enjeux de santé et les enjeux économiques de l'entreprise afin de construire des compromis efficaces pour la santé des travailleurs ;

- l'évaluation précise et multicritère de l'intervention ergonomique, tournée vers le futur.

5 Dans la première partie de la thèse, l'auteur insiste sur les enjeux économiques de la prévention des TMS à travers le coût direct des maladies professionnelles pour l'entreprise et les risques de précarisation du salarié. Il ajoute que la construction sociale de l'origine professionnelle de ce type de pathologie est progressive.

La deuxième partie de la thèse traite des marges de manœuvre.

«Les TMS sont le reflet d'un déficit de marges de manœuvre du salarié dans son travail, ce déficit individuel étant lui-même lié à un déficit de marges de manœuvre de nombreux acteurs dans l'entreprise » (p. 182).

7 L'intervention doit donc, pour être efficace, s'orienter vers une augmentation des marges de manœuvre de tous les acteurs et prévenir les TMS.

8 Les modélisations centrées autour des facteurs biomécaniques, physiques et individuels ont été élargies afin d'intégrer les facteurs psychosociaux, les facteurs organisationnels et le stress, même si la nature des relations possibles entre ces facteurs et les TMS n'est pas établie épidémiologiquement. Les TMS sont une atteinte globale de la santé des travailleurs. La santé est en relation forte avec la possibilité perçue par le travailleur d'être pour quelque chose dans ce qui lui arrive. C'est le développement du pouvoir d'agir des travailleurs qui est intéressant. Les TMS sont aussi un indicateur parmi d'autres de défaillance de l'organisation du travail - le reflet d'un déficit de marges de manœuvre. Selon l'auteur, ce déficit individuel est lui-même lié à un déficit plus large de marges de manœuvre des acteurs de l'entreprise.

Les parties 3 et 4 - Méthodes et Études de terrain - comportent six chapitres. La troisième partie porte sur les résultats de démarches participatives en ergonomie par rapport à la conduite de projets. Elle permet de définir les modalités d'évaluation de l'efficacité des projets. La quatrième partie montre que les recherches sur la pratique en ergonomie dans le champ de la prévention des TMS portent sur l'épidémiologie et l'expérimentation. Positionner la recherche sur la pratique comme un mode de production de connaissances pose des questions épistémologiques. Elle ne vise pas à prévoir l'activité des travailleurs mais davantage à anticiper, lors de la conception, les conditions de développement des marges de manœuvre.

10 La cinquième partie présente l'intervention ergonomique dans l'atelier de découpe de canard. Les enjeux de productivité sont un élément moteur de la décision de reconcevoir cet atelier. Plusieurs méthodes ont été élaborées : un questionnaire TMS pour l'évaluation avant et après des entretiens individuels avec le personnel 
d'encadrement, le suivi des résultats économiques du projet, les traces capitalisées durant le projet.

11 Les résultats montrent que l'augmentation des marges de manœuvre des opérateurs et de l'encadrement est un élément essentiel de la prévention des TMS. Ces marges de manœuvre sont essentiellement gagnées grâce à l'expertise ergonomique sur les nouvelles chaînes, la mise en place de la fonction de coordinateur de découpe, la participation des salariés au projet et la mise en place d'une formation adaptée. Plus précisément, l'intervention réalisée par l'auteur aboutit à plusieurs transformations du travail, notamment :

- la diminution de la cadence et l'augmentation de la distance entre deux canards sur la chaîne ont permis d'améliorer l'efficacité de la production ;

- la création des zones libres entre les postes favorise la production, notamment par la possibilité d'anticipation du travail ;

- l'aménagement de postes sur la chaîne et des modifications du processus de production ;

- la création d'un poste de coordinateur de découpe pour faire face au remplacement en cas d'absence et aider les opérateurs en cas de difficultés. Ce poste a permis aussi d'assurer les apprentissages.

12 Cette thèse, assez exemplaire, s'adresse à tous ceux concernés par la problématique des TMS, c'est-à-dire aux chercheurs des disciplines des relations santé-travail, aux acteurs de la prévention (médecins du travail, ergonomes, inspecteurs du travail, ingénieurs en hygiène et sécurité, etc.), enfin aux étudiants désirant acquérir une information ou une spécialisation dans ce domaine. Elle constitue un bon instrument d'intervention sur les questions de santé en lien avec l'efficacité et sur la conduite de projets de conception. Elle suggère des possibilités de formation à l'encadrement.

\section{AUTEURS}

\section{SANDRINE CAROLY}

Centre de Recherches en Innovation Socio-Technique et Organisation industrielle (CRISTO), Université Pierre Mendès France de Grenoble, BP47- 38040 Grenoble Cedex 9, Sandrine.Caroly@upmf-grenoble.fr 